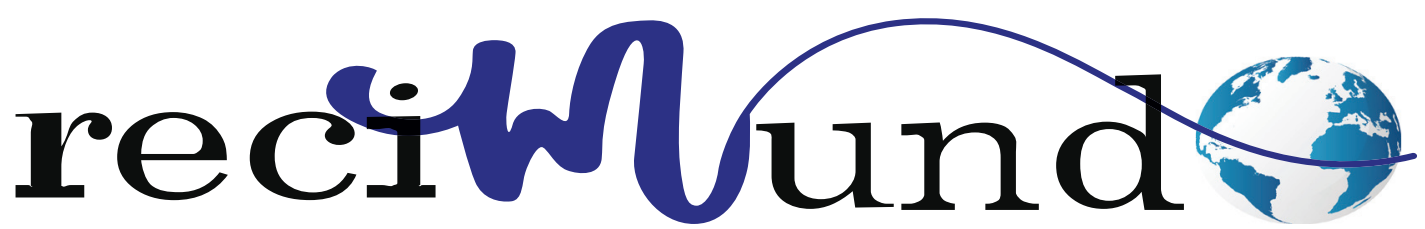

Revista Científica Mundo de la Investigación y el Conocimiento

DOI: 10.26820/recimundo/5.(Suple1).oct.2021.87-96

URL: https://recimundo.com/index.php/es/article/view/1335

EDITORIAL: Saberes del Conocimiento; Universidad Yachay Tech

REVISTA: RECIMUNDO

ISSN: 2588-073X

TIPO DE INVESTIGACIÓN: Artículo de revisión

CÓDIGO UNESCO: 3213.13 Estomatología

PAGINAS: 87-96

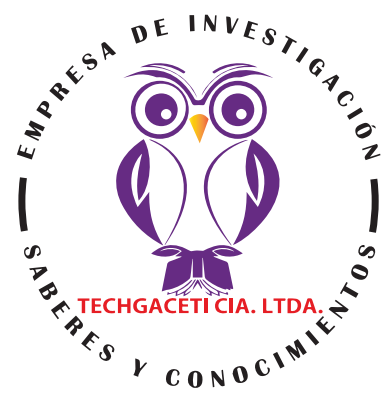

\title{
Apexificación en incisivos centrales superiores con biodentine
}

Apexification in upper central incisors with biodentine Apicificação em incisivos centrais superiores com biodentina

Jhannder Jhon González Yaguana'; Susana Patricia González Eras²;

Andrés Eugenio Barragán Ordoñez3; Marco Vinicio Carrión Sarmiento ${ }^{4}$

RECIBIDO: 02/09/2021 ACEPTADO: 20/09/2021 PUBLICADO: 30/10/2021

1. Odontólogo General; Universidad Nacional de Loja; Loja Ecuador; jhanderjhon@hotmail.com; (D) https://orcid. org/0000-0002-9513-5770

2. Odontóloga Especialista en Odontopediatría; Docente de la Carrera de Odontología de la Universidad Nacional de Loja; Loja, Ecuador; susana.p.gonzalez@unl.edu.ec; (D) https://orcid.org/0000-0001-9519-1150

3. Odontólogo Especialista en Ortodoncia; Docente de la Carrera de Odontología de la Universidad Nacional de Loja; Loja, Ecuador; andres.barragan@unl.edu.ec; (D) https://orcid.org/0000-0002-7884-9026

4. Odontólogo Posgradista en Especialización Ortodoncia Universidad Católica de Cuenca; markosog12@ gmail.com; (D) https://orcid.org/0000-0002-4383-3059

CORRESPONDENCIA

Jhannder Jhon González Yaguana

jhanderjhon@hotmail.com

Loja, Ecuador

๑) RECIMUNDO; Editorial Saberes del Conocimiento, 2021 


\section{RESUMEN}

La presente investigación corresponde a una revisión bibliográfica de tipo cualitativa, descriptiva y documental, el estudio fue realizado mediante criterios de exclusión e inclusión, para ello fueron analizados varios artículos científicos basados en apexificación, los cuales tuvieron como objetivo determinar la eficacia del Biodentine en la formación del cierre apical en los incisivos centrales superiores permanentes, además de identificar las propiedades de este material en relación con las estructuras dentales. El análisis utilizado fueron 26 artículos de tipo documental desde el año 2014 hasta el año 2020, revisiones bibliográficas, casos clínicos, los cuales fueron organizados en tablas diseñadas para el efecto. La apexificación la analizamos como un proceso que nos permite crear un ambiente dentro del conducto radicular y los tejidos periapicales luego de que se dé la muerte pulpar, lo cual permite la formación de una barrera calcifica a través del ápice abierto. Él Biodentine es un cemento de silicato de calcio que posee propiedades biocompatible y de bioactividad que al entrar en contacto directo con el tejido pulpar, induce al desarrollo de dentina reparativa lo cual logra mantener la vitalidad y función del tejido. Además, en relación con las estructuras dentales, la apexificación con Biodentine permite un sellado hermético de los túbulos dentinarios en dientes permanentes jóvenes, manteniendo las condiciones óptimas y correcto sellado apical.

Palabras clave: Ápice Del Diente, Apexificación, Necrosis Pulpar, Silicato de Calcio.

\section{ABSTRACT}

The present research corresponds to a qualitative, descriptive and documentary literature review, the study was carried out by criteria of exclusion and inclusion, for which several scientific articles based on apexification were analyzed, which aimed to determine the efficacy of Biodentine in the formation of apical closure in permanent upper central incisors, in addition to identifying the properties of this material in relation to dental structures. Method: 26 documentary articles were considered from 2014 to 2020, bibliographic reviews, clinical cases, which were organized in tables designed for the effect. Apexification is analyzed as a process that allows us to create an environment within the root canal and periapical tissues after pulp death occurs, which allows the formation of a calcifying barrier through the open apex. Biodentine is a calcium silicate cement that has biocompatible properties and bioactivity that, when in direct contact with the pulp tissue, induces the development of reparative dentin which manages to maintain the vitality and function of the tissue. In addition, in relation to dental structures, apexification with Biodentine allows an airtight sealing of the dentin tubules in permanent teeth Young permanent teeth, maintaining optimal conditions and correct apical sealing.

Keywords: Tooth Apex, Apexification, Pulp Necrosis, Calcium Silicate.

\section{ABSTRACT}

A presente pesquisa corresponde a uma revisão bibliográfica qualitativa, descritiva e documental, o estudo foi realizado por critérios de exclusão e inclusão, para os quais foram analisados diversos artigos científicos baseados em apicalização, os quais objetivaram determinar a eficácia do Biodentine na formação de apicais fechamento em incisivos centrais superiores permanentes, além de identificar as propriedades desse material em relação às estruturas dentais. Método: foram considerados 26 artigos documentais de 2014 a 2020, revisões bibliográficas, casos clínicos, os quais foram organizados em tabelas elaboradas para o efeito. A apicificação é analisada como um processo que nos permite criar um ambiente dentro do canal radicular e dos tecidos periapicais após a morte pulpar, o que permite a formação de uma barreira calcificante através do ápice aberto. O Biodentine é um cimento de silicato de cálcio que possui propriedades biocompatíveis e bioatividade que, ao entrar em contato direto com o tecido pulpar, induz o desenvolvimento de dentina reparadora que consegue manter a vitalidade e função do tecido. Além disso, em relação às estruturas dentais, a apicificação com Biodentine permite o selamento hermético dos túbulos dentinários dos dentes permanentes jovens, mantendo ótimas condições e correto selamento apical.

Keywords: Ápice Dentário, Apexificação, Necrose Pulpar, Silicato de Cálcio. 


\section{Introducción}

Dentro del proceso de desarrollo dentario está implicado la formación fisiológica de la raíz dental pudiendo verse afectada cuando existen injurias al tejido dentario provocando pulpitis irreversible sintomática o asintomática, provocándose un daño irreversible a la pulpa de manera que conduce a la necrosis del diente y a la irrupción de la formación fisiológica de la raíz dental, esto hace que este proceso se vea detenido, siendo necesario la intervención del odontólogo para guiar este desarrollo de formación de la raíz, cuyo proceso se lo conoce como "Apexificación" (Luzòn K.; Sánchez B.; González S.; Gahona D., 2020)

Coaguila 2014 (citado en Luzón Caigua et al., 2020, 136) la define como "un método para inducir el cierre apical en dientes no vitales con formación radicular incompleta y funciona formando una barrera mineralizada" dónde se realizan una serie de pasos en el tratamiento, teniendo como objetivo el uso de una solución para limpiar la cavidad pulpar y el conducto radicular para formar la barrera. Entre las opciones tradicionales de tratamiento se encuentra la apexificación con el uso de medicación intraconducto (MIC) de hidróxido de calcio, o la colocación del agregado de trióxido mineral (MTA) como barrera apical antes de obturar los conductos radiculares, pero no son las más precisas ya que estas opciones requieren un pronóstico a largo plazo debido a la dentina delgada en las paredes, sin embargo, existen estudios que comprueban que el Biodentine es un material bioactivo que contiene un gran nivel de efectividad como reparador de dentina, además, en la última década, la revascularización pulpar se ha considerado un nuevo método de tratamiento, promoviendo la formación de la raíz en dientes inmaduros (Cardoso A.; Herrera D.; Correia A.; Yuri J.; Farias T.; Soares A., 2016).
El tratamiento endodóntico de piezas dentales que presentan necrosis pulpar y ápice abierto suele ser un desafío para el profesional, debido a la anatomía radicular parcialmente formada, la pared radicular débil y la longitud radicular reducida (Ramírez W.; López F., 2018). Actualmente, disponemos para estos casos un material a base de silicato de calcio con biocompatibilidad y actividad biológica, que puede reemplazar la dentina dañada como es el Biodentine (Álvarez D.; Astudillo S.; Toral A., 2018); este biocerámico se considera una gran entidad cambiando el pronóstico de muchos casos con pronósticos reservados o casi imposibles de resolver (Kaur; Singh H.; Singh J.; Batra M.; Saini M., 2017).

El funcionamiento del cemento bioactivo (Biodentine) consiste en la penetración en los túbulos dentinarios abiertos, cristalizando o entrelazando los cristales con la dentina proporcionando así propiedades mecánicas (H., 2019). Además, este material está formulado con tecnología de cemento basada en MTA y, por lo tanto, afirma haber mejorado en ciertas propiedades, como la calidad física y el manejo, incluida su uso en una amplia gama de otras aplicaciones, como la reparación endodóntica y el recubrimiento pulpar en odontología restauradora (Kaur; Singh H.; Singh J.; Batra M.; Saini M., 2017). Por lo antes mencionado, el objetivo de este trabajo de investigación bibliográfica es determinar la eficacia de este nuevo material en el mercado odontológico, Biodentine, en la formación del cierre api$\mathrm{cal}$, identificando propiedades, funciones principales, así como su utilización, aportando y afianzando con nuevos conocimientos para la obtención de una nueva alternativa de tratamiento.

\section{Materiales y métodos}

\section{a. Diseño}

Esta revisión bibliográfica es de carácter: 
Cualitativa: ya que nos basamos en la descripción de las diferentes características del Biodentine que nos ayuda a solucionar problemas relacionados con la no formación del cierre apical. Además, esta revisión pretende analizar las situaciones en las que no se ha logrado el cierre apical y las circunstancias en las cuales se recomienda hacer una apexificación.

Descriptiva: ya que explica las características del Biodentine, su funcionamiento y cómo lo manipularemos. También, la técnica de apexificación y cómo será su adaptación en el proceso.

Documental: ya que se encarga de la revisión bibliográfica en fuentes que tengan similitud dentro de la ciencia odontológica, dando prioridad a las publicaciones recientes, aproximadamente hasta los últimos 5 años.

\section{b. Estrategia de búsqueda:}

La presente revisión bibliográfica fue cualitativa, descriptiva y documental, pues se realizaron análisis de diferentes artículos, las bases de datos utilizadas para la recolección de información acerca de este tema fueron: Dialnet, Scielo, Medigraphic, Medline y ScienceResearch.

Se desarrolló una estrategia de búsqueda con los términos: "Apexificación en incisivos centrales superiores permanentes con Biodentine", "BIODENTINE: una nueva alternativa en endodoncia para su aplicación en apexificación y perforaciones radiculares", Biodentine: un nuevo material en terapia pulpar.

\section{c. Criterios de inclusión:}

Todos los estudios primarios o revisiones bibliográficas en los que se abordó temas relacionados con apexificación en incisivos centrales superiores permanentes con Biodentine fueron tomados en cuenta.

Se midió de acuerdo a la calidad de las di- ferentes investigaciones, la pertinencia de los estudios y su continuidad en el tiempo, así como su capacidad real de responder a las dudas existentes acerca del tema en cuestión.

\section{d. Criterios de exclusión:}

Se ha realizado una revisión sistémica de documentos de revistas científicas dedicadas al estudio de apexificación con Biodentine. También se han consultado revisiones sistémicas y estudios científicos sobre el tema a tratar.

Se han localizado 32 artículos, pero se han excluido 6 que no fueron relevantes para el objetivo de la revisión. 


\section{Resultados}

\section{Eficacia}

\begin{tabular}{|c|c|c|c|c|}
\hline AUTOR / AÑO & TÍTULO & PROPIEDADES & FUNCIÓN & AUTOR / AÑO \\
\hline $\begin{array}{l}\text { (Barzuna M.; Tellez } \\
\text { A., 2018) }\end{array}$ & $\begin{array}{l}\text { Tapón apical con } \\
\text { biocerámicos: tra- } \\
\text { tamiento del ápice } \\
\text { abierto en una sola } \\
\text { cita. }\end{array}$ & $\begin{array}{l}\text { Paciente de } 11 \\
\text { años, traumatismo } \\
\text { a nivel de las pie- } \\
\text { zas 12, } 11 \text { y 22, } \\
\text { formación radicular } \\
\text { incompleta y ensan- } \\
\text { chamiento del es- } \\
\text { pacio del ligamento } \\
\text { vestibular. }\end{array}$ & $\begin{array}{l}\text { Apexificación sim- } \\
\text { plificada con Bio- } \\
\text { dentine. Observa- } \\
\text { ción por } 9 \text { meses. }\end{array}$ & $\begin{array}{l}\text { Buena reparación } \\
\text { periapical eviden- } \\
\text { ciada clínica y radio- } \\
\text { gráficamente a los } 9 \\
\text { meses con recupe- } \\
\text { ración de la pieza } \\
\text { del } 100 \%\end{array}$ \\
\hline (Caro A., 2017) & $\begin{array}{l}\text { Terapia de Re- } \\
\text { paración Pulpar } \\
\text { guiada en Dien- } \\
\text { te Inmaduro, en } \\
\text { Necrosis Pulpar, } \\
\text { realizando la Pre- } \\
\text { paración Biométri- } \\
\text { ca Completa con } \\
\text { Biodentine, Proto- } \\
\text { colo Universal de } \\
\text { Valparaíso. }\end{array}$ & $\begin{array}{l}\text { Paciente de } 18 \\
\text { años de edad, con } \\
\text { tratamiento dentro- } \\
\text { alveolar, pieza } 11 \\
\text { tracto fistuloso por } \\
\text { vestibular. }\end{array}$ & $\begin{array}{l}\text { Apexificación y se- } \\
\text { llado coronal con } \\
\text { Biodentine. }\end{array}$ & $\begin{array}{l}\text { Formación de teji- } \\
\text { dos de reparación, } \\
\text { cierre apical exitoso } \\
\text { después de } 5 \text { años } \\
\text { de control, se evi- } \\
\text { dencia en el informe } \\
\text { tomográficoo. Efica- } \\
\text { cia del } 100 \% \text {. }\end{array}$ \\
\hline $\begin{array}{l}\text { (López M; Rodrí- } \\
\text { guez A; Vegaa E, } \\
2016)\end{array}$ & $\begin{array}{l}\text { Tapón apical de } \\
\text { Biodentine en el } \\
\text { fragmento coronal } \\
\text { en fractura radicu- } \\
\text { lar horizontal. }\end{array}$ & $\begin{array}{l}\text { Paciente de } 35 \\
\text { años, presentó trau- } \\
\text { matismo a los } 16 \\
\text { años, Fracaso de } \\
\text { tratamiento de en- } \\
\text { dodoncia. Abceso } \\
\text { apical crónico, frac- } \\
\text { tura radicular hori- } \\
\text { zontal con despla- } \\
\text { zamiento del tercio } \\
\text { apical. }\end{array}$ & $\begin{array}{l}\text { Barrera apical con } \\
\text { Biodentine. }\end{array}$ & $\begin{array}{l}\text { Eficacia del } 70 \% \\
\text { debido a que el pa- } \\
\text { ciente se encuentra } \\
\text { en observación. }\end{array}$ \\
\hline $\begin{array}{l}\text { (Farías J; Garrido } \\
\text { M; Mercado M., } \\
2017 \text { ) }\end{array}$ & $\begin{array}{l}\text { Procedimientos } \\
\text { Endodónicos Re- } \\
\text { generativos con } \\
\text { Biodentine en } \\
\text { Dientes Perman- } \\
\text { tentes Jóvenes } \\
\text { con Periodontitis } \\
\text { Apical Asintomá- } \\
\text { tica tras Luxación } \\
\text { Extrusiva. }\end{array}$ & $\begin{array}{l}\text { Paciente de } 10 \text { años } \\
\text { de edad, traumatis- } \\
\text { mo en la pieza } 11 \\
\text { y } 21 \text { con formación } \\
\text { radicular incomple- } \\
\text { ta áreas radiolúci- } \\
\text { das pariapicale. }\end{array}$ & $\begin{array}{l}\text { Desinfección con } \\
\text { hipoclorito y medi- } \\
\text { cación intraconduc- } \\
\text { to. Formación del } \\
\text { coágulo y coloca- } \\
\text { ción del Biodentine. }\end{array}$ & $\begin{array}{l}\text { Apexificación exito- } \\
\text { sa, seguimiento por } \\
122 \text { meses del tra- } \\
\text { tamiento a través de } \\
\text { radiografías con una } \\
\text { eficacia demostrada } \\
\text { de un } 100 \% \text { a través } \\
\text { de estudios tomo- } \\
\text { gráficos. }\end{array}$ \\
\hline (M; Pabón E., 2020) & $\begin{array}{ll}\text { Tratamiento no } & \text { no } \\
\text { quirúrgico de } & \text { le- } \\
\text { siónperiapical de } & \text { gran tamaño. }\end{array}$ & $\begin{array}{l}\text { Paciente de } 21 \text { años } \\
\text { de edad, fístula en } \\
\text { la mucosa vestibu- } \\
\text { lar en la pieza } 2.1 \\
\text { postraumático. }\end{array}$ & $\begin{array}{l}\text { Desinfección con } \\
\text { clorhexidina y obtu- } \\
\text { ración del conducto } \\
\text { con Biodentine. }\end{array}$ & $\begin{array}{l}\text { Terapia de apexifi- } \\
\text { cación exitosa con } \\
3 \text { años de observa- } \\
\text { ción demostrada en } \\
\text { un } 100 \% \text { a través de } \\
\text { una tomografía y eli- } \\
\text { micación total de la } \\
\text { patología. }\end{array}$ \\
\hline $\begin{array}{l}\text { (Pradeep K.; Mo- } \\
\text { hata P.; Butula R., } \\
\text { 2018) }\end{array}$ & $\begin{array}{l}\text { Paciente con pul- } \\
\text { panecrótica y ápi- } \\
\text { ce abierto en la } \\
\text { pieza } 1.1\end{array}$ & $\begin{array}{l}\text { Paciente con pul- } \\
\text { panecrótica y ápice } \\
\text { abierto en la pieza } \\
1.1 .\end{array}$ & $\begin{array}{l}\text { Barrera apical con } \\
\text { Biodentine en una } \\
\text { sola cita. }\end{array}$ & $\begin{array}{l}\text { Eficacia del trata- } \\
\text { miento de un } 70 \% \\
\text { puesto que no se } \\
\text { evidencia segui- } \\
\text { miento del trata- } \\
\text { miento. }\end{array}$ \\
\hline
\end{tabular}


TO, M. V.

En la tabla antes expuesta, mediante el análisis de los 6 casos clínicos podemos determinar que en tratamientos de apexificación, fracturas y necrosis pulpar, utilizando el Bio- dentine, se obtuvo los resultados esperados con un control radiográfico y tomográfico cuya eficacia es del $90 \%$.

\section{Propiedades y Función}

\begin{tabular}{|c|c|c|c|c|}
\hline AUTOR / AÑO & TÍTULO & PROPIEDADES & FUNCIÓN & AUTOR / AÑO \\
\hline $\begin{array}{l}\text { (Barzuna M,; Tellez } \\
\text { A, 2018) }\end{array}$ & $\begin{array}{l}\text { Tapón apical con } \\
\text { biocerámicos: tra- } \\
\text { tamient del ápice } \\
\text { abierto en una sola } \\
\text { cita. }\end{array}$ & $\begin{array}{l}\text { Reparación Periapi- } \\
\text { cal. }\end{array}$ & $\begin{array}{l}\text { Formación de Ba- } \\
\text { rrera apical. }\end{array}$ & $\begin{array}{l}\text { (Barzuma M.; Tellez } \\
\text { A., 2018) }\end{array}$ \\
\hline (Caro .; 2017) & $\begin{array}{l}\text { Terapia de Repara- } \\
\text { ción Pulpar guiada } \\
\text { en Diente Inma- } \\
\text { duro, en Necrosis } \\
\text { Pulpar, realizando } \\
\text { Preparación Bio- } \\
\text { mecánica Comple- } \\
\text { ta con Biodentine, } \\
\text { Protocolo Universi- } \\
\text { dad de Valparaíso. }\end{array}$ & $\begin{array}{l}\text { Formación de tejido } \\
\text { de reparacón. }\end{array}$ & $\begin{array}{l}\text { Sellado coronario } \\
\text { Cierre Apical. }\end{array}$ & (Caro A.; 2017) \\
\hline $\begin{array}{l}\text { (López M.; Rodrí- } \\
\text { guez A.; Vega E, } \\
2016)\end{array}$ & $\begin{array}{l}\text { Tapón apical de } \\
\text { Biodentine en el } \\
\text { fragmento coronal } \\
\text { en Fractura radicu- } \\
\text { lar horizontal. }\end{array}$ & $\begin{array}{l}\text { Bioactividad Sella- } \\
\text { do Excelente. }\end{array}$ & $\begin{array}{l}\text { Formación de ba- } \\
\text { rrera Apical. }\end{array}$ & $\begin{array}{l}\text { (López M.; Rodri- } \\
\text { guez A.; Vega E, } \\
2016)\end{array}$ \\
\hline $\begin{array}{l}\text { (Farías J.; Garrido } \\
\text { M.; Mercado M., } \\
2017 \text { ) }\end{array}$ & 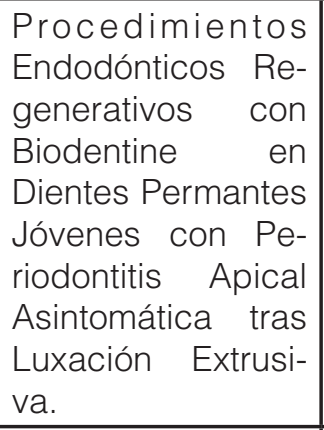 & Regenerativa. & Puente dentinario. & $\begin{array}{l}\text { (Farias J.; Garrido } \\
\text { M.; Mercado M., } \\
\text { 2017) }\end{array}$ \\
\hline $\begin{array}{l}\text { (Barzuna M.; Pabón } \\
\text { E., 2020) }\end{array}$ & $\begin{array}{l}\text { Tratamiento no qui- } \\
\text { rúrgico de lesión } \\
\text { periapical de gran } \\
\text { tamaño. }\end{array}$ & Bioactividad Fácil. & $\begin{array}{l}\text { Diferenciación de } \\
\text { células odontoblás- } \\
\text { ticas. Formación } \\
\text { completa de puente } \\
\text { dentinario. }\end{array}$ & $\begin{array}{l}\text { (Barzuna M.; Pabón } \\
\text { E., 2020) }\end{array}$ \\
\hline $\begin{array}{l}\text { (Pradeep K.; Mo- } \\
\text { hata P.; Butula R., } \\
2018 \text { ) }\end{array}$ & $\begin{array}{lr}\text { Biodentine: } & \text { novel } \\
\text { endodontic } & \text { mate- } \\
\text { rial for single } & \text { step } \\
\text { apexificatión: } & \text { a } \\
\text { case report. } & \end{array}$ & $\begin{array}{l}\text { Solubilidad Fragua- } \\
\text { do corto Mineraliza- } \\
\text { ción Biométrica. }\end{array}$ & Tapón Apical. & $\begin{array}{l}\text { (Pradeep K.; Mohata } \\
\text { P.; Butula R., 2018) }\end{array}$ \\
\hline
\end{tabular}

En la tabla antes mencionada, en las que se analiza las propiedades y función del Biodentine, en base a los artículos revisados, podemos determinar que es un material de fácil manejo que induce a la formación de tejidos de reparación con un excelente sellado, que permite la formación del cierre apical, debido a que es un material compatible con la dentina y a la pulpa. 


\section{Discusión}

Los objetivos de la presente revisión bibliográfica es determinar las propiedades, efectividad y la función del Biodentine en la terapia de apexificación en dientes cuyo diagnóstico es necrosis pulpar.

Con el transcurrir del tiempo se han ido desarrollando diversos materiales aplicados en el tratamiento de apexificación, como es el BIODENTINE utilizado como un sustituto dentinario bioactivo que posee propiedades mecánicas similares a la dentina sana y puede reemplazarla tanto a nivel coronario como a nivel radicular.

Una maravillosa ventaja de este material es su tiempo de fraguado final que es de $10 \mathrm{a}$ 12 minutos, con respecto a otros cementos se debe al cambio en el tamaño de las partículas, puesto que a mayor superficie es menor el tiempo de fraguado y la adición de cloruro de calcio al vehículo, que acelera la reacción y disminuye el contenido líquido.

Por lo tanto, he desarrollado una revisión bibliográfica acerca de diversos casos clínicos haciendo alusión al uso del Biodentine en la apexificación de incisivos centrales superiores. Según el reporte de caso denominado: "Tapón apical con biocerámicos. Tratamiento del ápice abierto en una sola cita", nos señalan (Barzuma M.; Tellez A., 2018) que la aplicación de Biodentine en el tratamiento de Apexificación se ha considerado tratamiento eficaz para piezas inmaduras necróticas, además, permite reducir los tiempos operatorios y así mejorar la calidad de vida del paciente, demostrando resultados positivos en cuanto a la reparación periapical, tanto clínica como radiográficamente en los controles periódicos.

(Caro A., 2017) en su reporte de caso: "Terapia guiada en diente inmaduro, en necrosis pulpar, realizando preparación biomecánica completa con Biodentine, protocolo Universidad de Valparaiso" refleja la problemática existente en piezas dentales con ápice abierto con paredes radiculares no desarrolladas completamente y que se asocian a una lesión periapical. y cómo resolver este problema, aplicando el uso de Biodentine para la inducción del cierre apical, por su capacidad de formación de tejido de reparación, aportando ventajas en el presente caso, considerándose exitoso después de cinco años de control, en un informe tomográfico se observó zonas de sanado fibroóseo, ausencia de fracturas radiculares, leve incremento de la longitud radicular, disminución de la zona radiolúcida y la formación del cierre apical.

Las frecuencias de las fracturas radiculares son responsables del $1 \%$ de la pérdida de la dentición permanente según, (López M.; Rodríguez A.; Vega E, 2016) en su estudio: "Tapón apical de Biodentine en el fragmento coronal en fractura radicular horizontal" en el cual indica que el éxito o buen pronóstico de la fractura radicular horizontal en dientes con ápices abiertos la colocación de una barrera apical (Biodentine). Obteniendo un resultado exitoso en la reparación de esta fractura en el cual el material anteriormente mencionado ofrece bioactividad y propiedades de sellado excelente.

En el estudio de Peralta, A (2019) "Manejo de complicación post-traumática dental" nos menciona la aplicación de un biocerámico para inducir a la apexificación, además del empleo de medicación intraconducto como lo fue el hidróxido de calcio, logrando así una técnica adecuada para tratar la patología. Ayudados de los exámenes tomográficos se pudo comprobar que la colocación del Biodentine en el tercio apical logró inducir al cierre apical y la continuidad del espacio del ligamento periodontal a lo largo de toda la raíz.

En este caso clínico: "Procedimientos endodónticos regenerativos con Biodentine en dientes permanentes jóvenes con periodontitis apical asintomática tras luxación

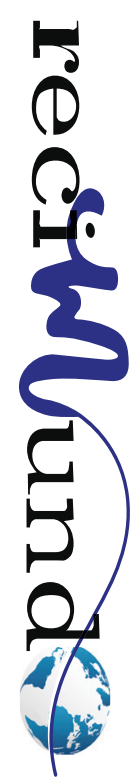


extrusiva" tuvo éxito el uso del Biodentine, considerado un material adecuado para una terapia de apexificación. Según (Farias J.; Garrido M.; Mercado M., 2017) la consideraron como un material regenerativo, porque inducen a la diferenciación de células odontoblásticas y a la proliferación de otras células. Se hace el seguimiento constante durante un año lo cual el paciente permanece asintomático, las lesiones en regresión y se logra la formación de puente dentinario en zona periapical. Por lo tanto, (Barzuna M.; Pabón E., 2020) en este estudio obtiene beneficios mediante la técnica usada, obturando el conducto radicular completamente con Biodentine. En los controles clínicos y tomográficos se hace un análisis comparativo en cada cita, observado un cambio notorio de la formación ósea.

El manejo de un diente inmaduro con necrosis pulpar y la patología periapical plantea un gran desafío en la práctica endodóntica. Las opciones de tratamiento comprenden el procedimiento convencional de apexificación con y sin barreras apicales. En la investigación "BIODENTINE: NOVEL ENDODONTIC MATERIAL FOR SINGLE STEP APEXIFICATION" autoría de (Pradeep k.; Mohata P.; Butula R., 2018) propone la ejecución de un tratamiento de apexificación en una sola visita. El uso de Biodentine, puede ser utilizado como una alternativa eficaz al MTA, incluso en el paciente mayor, ya que este material posee un gran potencial en el manejo de un diente con un ápice abierto, particularmente en su capacidad para lograr mineralización biomimética. (Pradeep k.; Mohata P.; Butula R., 2018) llegaron a la conclusión que el Biodentine induce la regeneración de la dentina al estimular las células progenitoras de la pulpa en el proceso de apexificación.

\section{Conclusiones}

Podemos concluir que la eficiencia del Biodentine se debe a las diferentes propiedades similares a la dentina como a la reparación apical, formación del tejido de reparación, bioactividad, excelente sellado, fácil manejo, fraguado corto y mineralización biomimética.

De acuerdo a la eficiencia, el Biodentine, según las revisiones de la literatura se han encontrado que en la mayor parte de los casos clínicos ha dado una alta resistencia mecánica, además, su composición de silicato tricálcico también se definen como una fuente de hidroxiapatita cuando están en contacto con el fluido tisular sintético, por lo que tiene una excelente biocompatibilidad con la dentina ayudando también a la formación de dentina reparadora y tejido duro.

El Biodentine a pesar de ser un material nuevo resulta muy innovador, dando resultados positivos en los casos estudiados, presentando ciertas ventajas en comparación a otros materiales como un menor costo que el de sus competidores, su tiempo de fraguado superior, su alta resistencia mecánica, la biocompatibilidad y no toxicidad, así como también posee también actividad antibacteriana.

\section{Agradecimiento}

Agradezco a Dios y a la Virgen Santísima por haberme permitido culminar mi carrera universitaria y también por haberme permitido tener una familia maravillosa que me ha brindado su apoyo incondicional, su amor y su ejemplo de superación, humildad y sacrificio, de manera muy especial a mis padres queridos, que hicieron todo lo posible para que el día de hoy uno de mis más grandes sueños se haya cumplido.

Agradezco al docente, Dr. Andrés Barragán Ordóñez por que ha sabido guiarme durante toda mi trayectoria universitaria a través de su experiencia y dedicación para llegar a ser una gran profesional, además de un docente, siempre ha sido un amigo que me ha apoyado en las buenas y en las malas, además me supo abrir las puertas de su 
consultorio para adquirir experiencia día a día en el ámbito clínico.

Agradezco a mi directora de tesis, Dra. Susana González, por su tiempo y su paciencia dedicada durante la elaboración de este proyecto.

\section{Bibliografía}

1. Álvarez D.; Astudillo S.; Toral A. (2018). BIODENTINE COMO RECUBRIMIENTO PULPAR DIRECTO. REPORTE DE CASO CLINICO. Revista Evid. Odontol. Clinic., 49-55. Obtenido de https://www. researchgate.net/publication/335482703_BIODENTINE_COMO_RECUBRIMIENTO_PULPAR_ DIRECTO_REPORTE_DE_CASO_CLINICO

2. Barzuma M.; Tellez A. (abril de 2018). Tapón apical con biocerámicos: tratamiento del ápice abierto en una cita. Odontología Vital, 33-38. Obtenido de https://www.google.com.mx/url?$s a=t \& r c t=j \& q=\& e s r c=s \&$ source $=$ web\& $c d=\& v e-$ $\mathrm{d}=2$ ahUKEwiOzoXE6YPXAhXUFIkFHUDXCFcQFjADegQIDxAD\&url=https\%3A\%2F\%2Fwww. scielo.sa.cr\%2Fpdf\%2Fodov\%2Fn29\%2F1659-0775-odov-29-33. pdf\&usg=AOvVaw2QyKJQbovPb/Ti7ZqmvM×5

3. Barzuna M.; Pabón E. (2020). Tratamiento no quirurgico de lesión periapical de gran tamaño: reporte de un caso. Odontología Vital, 29-38. Obtenido de https://www.scielo.sa.cr/scielo.php?script=sci_arttext\&pid=S1659-07752020000100029\&In$\mathrm{g}=\mathrm{en} \& \mathrm{nrm}=\mathrm{iso}$

4. Blázquez P.; Riolobos M. (2019). Técnica de revascularización en odontopediatría paso a paso. A propósito de un caso. Revista Odontopediatría Latinoamericana, 9(2). Obtenido dehttps://www. revistaodontopediatria.org/index.php/alop/article/ view/174

5. Cardoso A.; Herrera D.; Correia A.; Yuri J.; Farias T.; Soares A. (Diciembre de 2016). Alternativas clínicas para el tratamiento de dientes traumatizados con rizogénesis incompleta; una revisiòn actualizada. Estomatológica Herediana, 272. Obtenido de http://www.scielo.org.pe/scielo.php?script=sci_arttext\&pid=\$1019-43552016000400010

6. Caro A. (2017). Terapia de Reparación Pulpar guiada en Diente Inmaduro, en Necrosis Pulpar,realizando Preparación Biomecánica Completa con Biodentine, Protocolo Universidad de Valparaíso. Canal Abierto, 30-35. Obtenido de https://www.google.com.mx/url?$s a=t \& r c t=j \& q=\& e s r c=s \&$ source $=$ web \&cd $=\& v e-$ $\mathrm{d}=2$ ahUKEwiOzoXE6YPXAhXUFIkFHUDXCFcQFjASegQICRAD\&url=https\%3A\%2F\%2Fwww.
socendochile.cl\%2Frevista.php\%3Fid\%3D36.pdf\&usg=AOvVaw2gl|DnNzmM_YIJqjozET2_

7. Coaguilla H.; Denegri A. (2014). Uso de barreras apicales y apexificación en endodoncia. Revista Estomatològica Herediana, 24(2), 120-125. Obtenido de http://www.redalyc.org/articulo. oa?id=421539381009

8. Darak P.; Likhitkar M.; Goenka S.; Kumar A.; Madale P.; Kelode A. (2020). Comparative evaluation of fracture resistance of simulated immature teeth and its effect on single visit apexification versus complete obturation using MTA and Biodentine. Journal of Family Medicine and Primary Care, 9, 2011-2015. Obtenido de https://www.jfmpc.com/ article. asp? issn =2249-4863; year =2020; volume $=9$; issue $=4$; spage $=2011$; epage $=2015$; aulast $=-$ Darak

9. Farias J.; Garrido M.; Mercado M. (2017). Procedimientos Endodónticos Regenerativos con Biodentine en dientes permanentes jóvenes con periodontitis apical asintomática tras luxación extrusiva. Canal Abierto, 5-8. Obtenido de https:// www. google.com. $m \times / u r l$ ? sa $=t \& r c t=j \& q=\& e s-$ $r c=s \&$ source $=$ web\& $c d=\& v e d=2$ ahUKEwiO ZoXE6YPXAhXUFIkFHUDXCFCQFjASegQICRAD\&url=https\%3A\%2F\%2Fwww. socendochile.cl\%2Frevista.php\%3Fid\%3D36.pdf\&usg=AOvVaw2gIIDnNzmM_YIJajozET2 _

10. Gurría A.; Vilchis S.; Rodríguez A. (2019). Uso de biodentine como alternativa de recubrimiento pulpar. Revista méxicana de estomatología, 32-40. Obtenido de https://www.remexesto.com/index. php/remexesto/article/view/288/551

11. H., M. (10 de Septiembre de 2019). Repositorio Universidad de Guayaquil. Obtenido de Protección pulpa directa con BIODENTINE: http://repositorio.ug.edu.ec/handle/redug/44289

12. Hargreaves K.; Berman L. (2016). Cohen. Vías de la pulpa. Barcelona, España: Elsevier. Obtenido de https://www.laleo.com/cohen-vias-de-la-pulpa-p-20452.html

13. Hervoso M.; Barzuna M. (julio de 2017). UNA NUEVA ALTERNATIVA PARA DIENTES INMADUROS CON PULPA NECRÓTICA: APICOFORMACIÓN USANDO HIDRÓXIDO DE CALCIO CON YODOFORMO Y UN BIOCERAMICO. Revista Cientifica Odontológica, 25-31. Obtenido de http:// www. redalyc. org/articulo.oa?id=324255459005

14. Hincapié S.; Valerio A. (2015). Biodentine: Un nuevo material en terapia pulpar. Univ.Odontol., 69-76. Obtenido de https://www.google.com/ url?sa $=t \& r c t=j \& q=\& e s r c=s \&$ source $=$ web \&c$\mathrm{d}=\& \mathrm{cad}=\mathrm{rja} \&$ uact $=8 \& \mathrm{ved}=2 \mathrm{ahUKEwih7YLIqI}-$ 
TO, M. V.

TxAhXkhOAKHTNACmEQFjADegQIAhAD\&ur$\mathrm{I}=\mathrm{https} \% 3 \mathrm{~A} \% 2 \mathrm{~F} \% 2 \mathrm{Frevistas}$. javeriana.edu. co\%2Findex.php\%2FrevUnivOdontologica\%2Farticle\%2Fview\%2F16040\%2F12839\&usg=AOvVaw3K9CHq

15. Kaur; Singh H.; Singh J.; Batra M.; Saini M. (2017). MTA versus Biodentine: Review of Literature with a Comparative Analysis. Journal of Clinical and Diagnostic Research., 1-4. doi: 10.7860/ JCDR/2017/25840.10374

16. López M.; Rodríguez A.; Vega E. (2016). Tapón apical de Biodentine en el fragmento coronal en fractura radicular horizontal. Revista mexicana de estomatología, 186-187. Obtenido de https://www.google.com/url?sa=t\&rct=j\&q=\&es$r c=s \&$ source $=$ web \&cd $=\& c a d=r j a \&$ uact $=8 \& v e-$ $\mathrm{d}=2$ ahUKEwjRvc7nu4TXAhXyQ98KHW33AIsQFjAAegQIAhAD\&url=https\%3A\%2F\%2Fwww. remexesto.com\%2Findex.php\%2Fremexesto\%2Farticle\%2Fview\%2F101\%2F185\&usg=AOvVaw0-tILmAkXcGO3aizFwMrEq

17. Luzòn K.; Sánchez B.; González S.; Gahona D. (15 de Octubre de 2020). Apicoformaciòn en dientes necróticos. Revisiòn narrativa. Recimundo, 134-143. doi:10.26820/recimundo/4.(4).octubre.2020.134-143

18. Méndez V.; Madrid K.; Amador E.; Silva D.; Oliva R. (2014). Revascularización en dientes permanentes. ADM, 110-113. Obtenido de https://www. medigraphic.com/cgi-bin/new/resumen.cgi?IDARTICULO=50392
19. Pradeep k.; Mohata P.; Butula R. (2018). Biodentine: novel endodontic material for single step apexification: A Case report. SADA, 452-455. Obtenido de https://www.google.com/url?sa=t\&rct=j\&q=\&es$r c=s \&$ source $=$ web \& $c d=\& c a d=r j a \& u a c t=8 \& v e-$ $\mathrm{d}=2$ ahUKEwjx14af4YTxAhUyTTABHevRBYMQFjAAegQIBBAD\&url=http\%3A\%2F\%2Fwww. scielo.org.za\%2Fscielo.php\%3Fscript\%3Dsci_arttext\%26pid\%3DS0011-85162018000700006\%26Ing\%3Den\%26nrm\%3Diso\%2

20. Ramírez W.; López F. (2018). Apexificación con Biodentine. Revista Mexicana de Estomatología, 5(1), 5. Obtenido de https://www.remexesto.com/ index.php/remexesto/article/view/179/361

21. Vilches F.; Hernández A.; García O.; Rodríguez Y.; Pérez M.; Liriano R. (2017). Caracterización de las enfermedades pulpares en pacientes pertenecientes al Área II del municipio Cienfuegos. Medisur, 15(3), 328. Obtenido de http://www.medisur. sld.cu/index.php/medisur/article/view/3580

22. Vilchis S.; Gurria A.; Rodriguez A.; Treviño R. (2018). Necrosis pulpar con lesión periapical. Revista Mexicana de Estomatología, 5(2), 18-22. Obtenido de http://remexesto.com

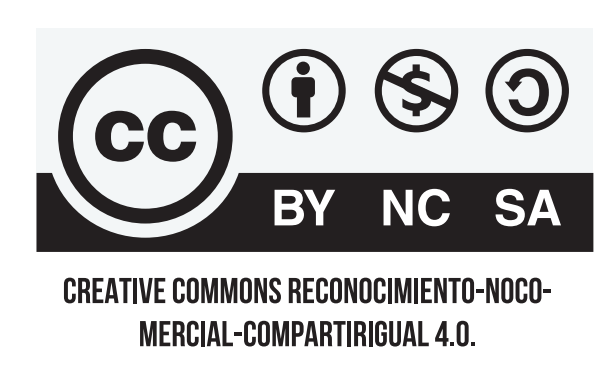

\section{CITAR ESTE ARTICULO:}

González Yaguana, J. J., González Eras, S. P., Barragán Ordoñez, A. E., \& Carrión Sarmiento, M. V. (2021). Apexificación en incisivos centrales superiores con Biodentine. RECIMUNDO, 5(1 (Suple), 87-96. https://doi.org/10.26820/recimundo/5.(Suple1).oct.2021.87-96 